\title{
Sign Language Gesture Recognition System for Hearing Impaired People
}

\author{
Uttam Prabhu Dessai \\ Department of Electronics \& Telecommunication \\ Goa College of Engineering \\ Ponda, India
}

\author{
Amita Dessai \\ Department of Electronics \& Telecommunication \\ Goa College of Engineering \\ Ponda, India
}

\begin{abstract}
The hand gesture is one of a powerful means of communication among human. Sign language is an essential and natural expressive mean of communication for the deaf people. American sign language helps the hearing-impaired people to communicate with the world as well as the computer. This paper proposes a technique for the recognition of American alphabet sign language. The technique of image processing, that is singular value decomposition (SVD), is applied in order to extract characteristics of the hand images performing individual alphabet of American sign language. The decomposed singular values extracted from the image are used to classify the image into one of the ASL alphabets. These SV features are then applied to SVM classifier for gesture recognition. The totals of 26 American sign language gestures are used in the experiments. This procedure is executed on a database with a total of 899 images of static American sign language gestures taken under different background and lighting conditions. The recognition rate of the proposed technique at about $74 \%$ is achieved.
\end{abstract}

\section{Keywords}

American sign language, Singular value decomposition , Support vector machine.

\section{INTRODUCTION}

We depend on verbal communication and body language for our daily life communication. The speech and hearing-impaired people can only on gestures to communicate. Gestures are defined as the motion of the body that is intended to communicate with other agents. For a successful communication sender and the receiver must have the same set of information for a particular gesture. As per the context of this project, gesture is defined as an expressive movement of hands which has a particular message, to be communicated precisely between a sender and a receiver. Hand gestures can be classified in two categories: static and dynamic. A static hand gesture is a particular hand configuration and pose, represented by a single image. A dynamic hand gesture is a moving gesture, which is represented by a sequence of images This project focuses on the recognition of static images.

American Sign Language (ASL) is a natural language which is a predominant sign language of speech and hearing-impaired people in the United States and most of Canada. Besides America, dialects of ASL are used in many countries around the world, including much of West Africa and parts of Southeast Asia.

That is why, a fast and accurate system is required to make this human computer interaction (HCI) more convenient to the deaf and dumb people. There are two fundamental techniques to gather the data identified with motion acknowledgment. First is the gadget-based estimation which does it with the help of information gloves and sensors. While the second way is the vision-based procedure, which covers the hand signer.

Following is a list of papers which were referred for this project. The research till now has been limited to small systems which are able to recognize minimal number of gestures.

Soumya and Muzameel developed [1] a gesture recognition system to identify and classify the images of Bharatnatya mudras using the shape parameters like area, centroid, eccentricity, major axis length etc. and artificial neural network (ANN). Sruthi and Thamizharasi proposed [2] an American sign language interpreter using the concepts of principal component analysis (PCA) and $\mathrm{K}$ nearest neighbor classifier. Tin Hninn Maung developed [3] a real time hand tracking and gesture recognition system using orientation histograms (HOG) and a perceptron network. Ahsan, Ibrahimy and Khalifa proposed [4] a hand motion detection system by utilizing the electromyography (EMG) signals and a back-propagating network with Levenberg-Marquardt algorithm. Wang, Syu, Li and Yang [5] employed symmetric mask based discrete wavelength transform to reduce the computational time by $66 \%$. Ankita Saxena, Deepak Jain and Ananya Singhal developed [6] hand gesture recognition system for an android device using sobel algorithm and back propagation neural network. Rahat Yasir and Riasat Azim Khan [7] proposed a twohanded hand gesture recognition system for Bangla sign language using linear discriminant analysis (LDA) and artificial neural network. Khan Mohammed and Razib Chandra Deb [8] utilized hidden markov model to recognize gestures. Some other researchers used techniques like edge detection, cross correlation and even fusion of neural network with different architectures of fuzzy technique.

The process has four stages, namely, image acquisition, image pre-processing, feature extraction and classification.

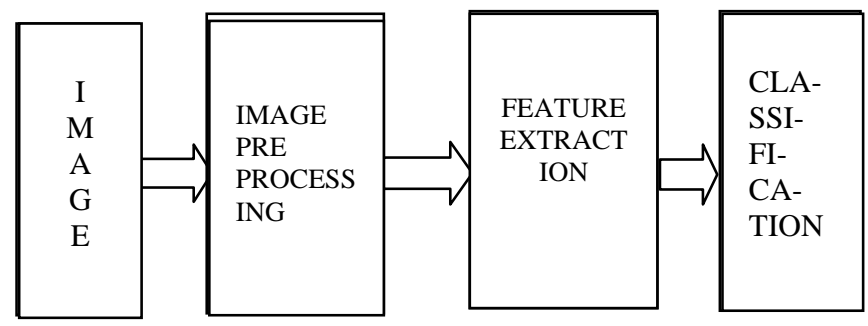

Figure 1: Block Diagram

The block diagram of the system is shown in the above figure 1. Here, we don't need any image acquisition device as a database named 'hand gesture recognition (HGR) ${ }^{[1]}$ is used. The database ${ }^{[1]}$ contains 899 original RGB images (jpg files) of 25 ASL gestures by 12 individuals. The image dimensions vary from 174x131 up to $640 x 480$.The images are taken in uncontrolled lighting conditions and have uncontrolled background. The images from this database ${ }^{[1]}$ are the input to the pre-processing stage. A number of pre-processing steps are carried out in the second 
stage. In the third stage, singular value decomposition (SVD) is used to extract features from the pre-processed image. In the final stage, the singular values are subjected to a Support vector machine (SVM) classifier.

MATLAB software is used for the development of this project. The reason behind selects MATLAB is its toolboxes. Toolboxes are comprehensive collections of $\mathrm{M}$ files that extend the MATLAB environment to solve particular classes of problems. It includes image processing, neural networks and classification learner toolboxes.

This paper is organized into six sections. Section II, III and IV elaborates on pre-processing, feature extraction and classification techniques respectively. While section V and VI discusses the results and the conclusion. Future work is specified in section VII.

\section{IMAGE PRE-PROCESSING}

The images of gestures in the HGR database ${ }^{[1]}$ are input to this hand gesture recognition system. While processing these images, every image is converted into matrix form. This matrix of a RGB image is a matrix of very high order having elements of different values. Working with such high values is very difficult, time consuming and will increase the computational time. Hence, the input RGB image is resized to $150 \times 150$ and then converted into a grayscale image. Resizing is done because image dimension of the input images varies from 174x131 up to $640 x 480$.The matrix of a grayscaled image has values from 0 to 255 only which makes it easy to work with. These images are then subjected to thresholding to separate the region of interest (hand) from the surroundings. Here, global thresholding is used for this purpose. As the name suggests, the intensity level (threshold) is global to the image, i.e. same threshold value is used to distinguish between the ROI and the background noise. This thresholded image is the input to the feature extraction stage.

\section{FEATURE EXTRACTION}

This section describes the feature extraction procedure implemented in this project. Here, singular value matrices are obtained by decomposition of the pre-processed image matrix. Singular value decomposition (SVD) is a popular and widely used technique for feature extraction. It also comes with an additional advantage of dimensionality reduction. SVD is a numerical technique used to diagnose matrices in numerical analysis. The singular value decomposition of a matrix $\mathrm{P}$ is the factorization of $\mathrm{P}$ into the product of three matrice $\mathrm{P}=\mathrm{UxDxV} \mathrm{V}^{\mathrm{T}}$ where the columns of $\mathrm{U}$ and $\mathrm{V}$ are orthogonal and the matrix $\mathrm{D}$ is diagonal with positive real entries. The main and important properties of SVD which are relevant from the viewpoint of image processing applications are that: when a small perturbation is added to an image, the singular values (SVs) of an image have very good stability, its SVs do not change significantly; and secondly, SVs represent intrinsic algebraic image properties. SVD is that it can be performed on any real $(m, n)$ matrix [8]. A matrix $\mathrm{P}$ with $\mathrm{m}$ rows and $\mathrm{n}$ columns, with rank $\mathrm{r}$ and $\mathrm{r} \leq \mathrm{n} \leq \mathrm{m}$. Then the $\mathrm{P}$ can be factorized into three matrices: $\mathrm{P}=\mathrm{UxDxV}^{\mathrm{T}}$ as where $\mathrm{U}$ and $\mathrm{V}$ are orthogonal matrices, and $\mathrm{S}$ is a diagonal matrix.

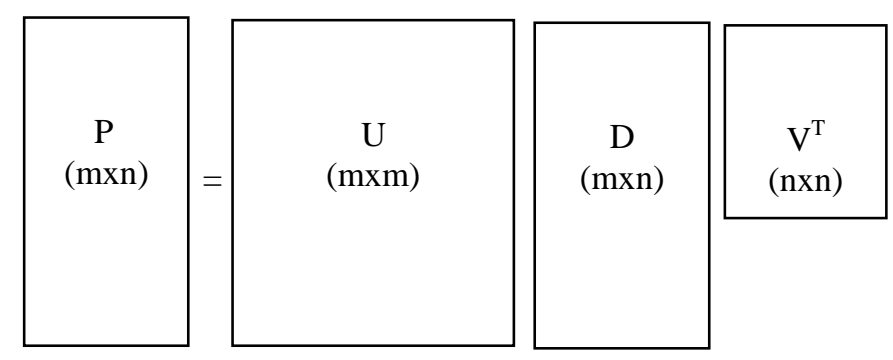

Figure 2: Singular value decomposition

The above figure shows the decomposition of input image $\mathrm{P}$ into matrix $U, D$ and $V^{T}$. The matrix $U$ is sometimes referred to as left singular vector matrix while matrix $\mathrm{V}^{\mathrm{T}}$ is referred to as right singular vector matrix. SVD provides the user with the best axis to project the data on i.e. the sum of squares of projection error is minimal. This algorithm is developed for a variety of applications. The traditional and most popular use of SVD is in Principal Component Analysis (PCA). Applications that use the SVD are computing the pseudoinverse, least squares fitting of data, multivariable control, matrix approximation, and determining the rank, range and null space of a matrix. These decomposed singular vectors are then used for classification of the gestures. SVD is one of the simplest feature extraction techniques which gives a lower dimensional picture.

\section{CLASSIFICATION}

The classification of the ASL hand gestures is done by implementing the support vector machine (SVM) classifier. The decomposed singular values from the previous stage are used to classify the hand gestures. The SVM classier effectively and efficiently classifies the hand gestures based on the singular value matrices. SVM is a supervised learning algorithm wherein each data item is plotted in a nxn dimensional space ( $\mathrm{n}$ is the number of features). Here the value of each feature is the value of that particular coordinate. Then, it determines a hyperplane which helps in segregation of the data items into different classes. The best choice will be the hyperplane that leaves the maximum margin from the classes involved. The margin is determined by its distance from the support vectors. SVM works the best when there is a clear margin of separation between the classes and is also very effective in high dimensional spaces. The SVM classifier is used in a number of applications like face detection, handwriting recognition, bioinformatics etc. Its algorithm makes SVM an opt choice for this hand gesture recognition project having 26 different classes.

\section{EXPERIMENTAL RESULTS}

This section discusses the various results of the different processes involved in this hand gesture detection project. Out of 899 images from the database ${ }^{[1]}$, there are 35 images of a particular gesture with different orientation. The results are obtained by testing the implemented system with the images of ASL hand gestures in the database. 


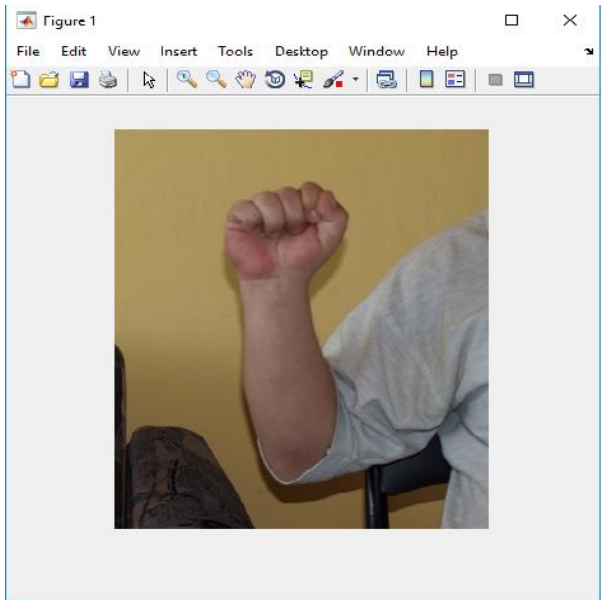

Figure 2: Original RGB image from database ${ }^{[1]}$

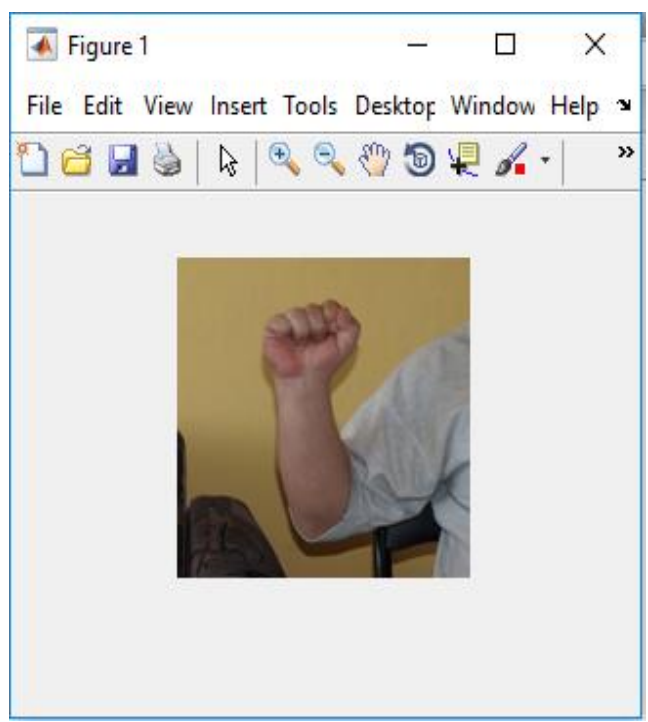

Figure 3: Resized RGB image

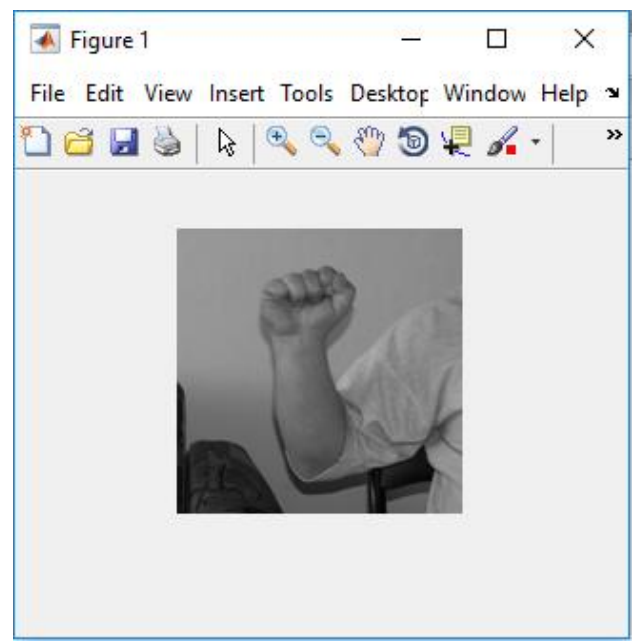

Figure 3: Grayscale image

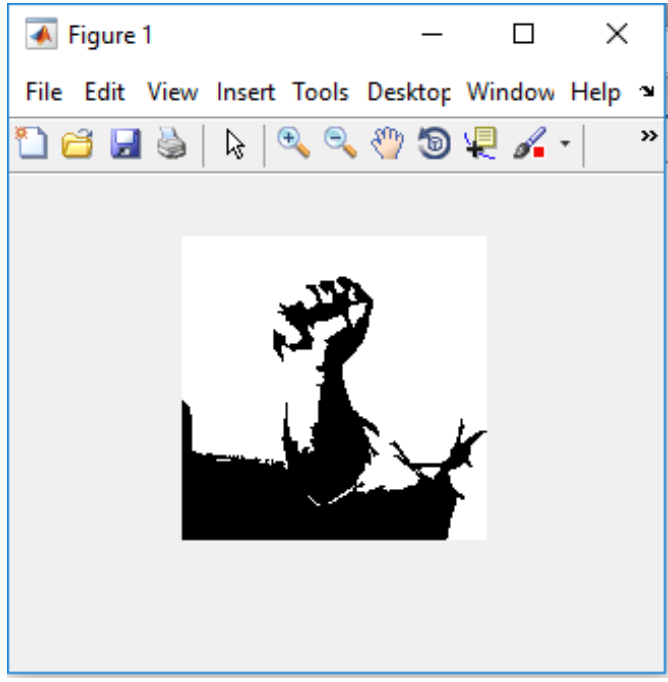

Figure 4: Thresholded image

According to the given input and obtained outputs, the accuracy of the system is calculated. As the system is under experiments, a $100 \%$ accuracy couldn't be achieved. An accuracy of $74 \%$ is obtained from this system.

\section{CONCLUSION}

The aim of this project is to provide the speech and hearingimpaired people with a fast and accurate hand gesture recognition system which can help them in their daily life communication. The system implemented here obtained an accuracy of $74 \%$ which can be increased further. The images with light as well as dark background and clicked in uncontrolled lighting conditions are involved in this project.

\section{FUTURE WORK}

By properly studying the limitations and shortcomings of this implemented processes like feature extraction and classification, a more accurate hand gesture recognition system can be developed. A larger database will help the classification process to achieve better accuracy. Features which are most effective in differentiating between the gestures need to be extracted. Effective classification methods like convolutional neural network (CNN), deep learning need to be implemented to meet the target.

\section{REFERENCES}

[1] HGR1 database, http://sun.aei.polsl.pl/ mkawulok/gestures/

[2] C.V Soumya, Muzameel Ahmed," Artificial Neural Network based Identification and Classification of images of Bharatanatyam Gestures", International Conference on Innovative Mechanisms for Industry Applications 2017.

[3] Sruthi Upendran, Thamizharasi A, "American Sign Language Interpreter for Deaf and Dumb Individuals", International Conference on Control, Instrumentation, Communication and Computational Technologies 2014.

[4] Tin Hninn Hninn Maung," Real Time Tracking and Gesture Recognition System using Neural Networks", International Journal of Computer, Electrical, Automation, Control and Information Engineering 2009.

[5] Md R Ahsan, Muhammed I. Ibrahimy, Othman O. Khalifa,' Hand motion detection from EMG signals by using ANN based classifier for human computer interaction", International Conference on Innovative Mechanisms for Industry Applications 2011. 
[6] Yuh Rau Wang, Jia Liang Syu, Hsin Ting Li, Ling Yang,", Fast hand detection and gesture recognition", International conference on machine learning and cybernetics 2015.

[7] Ankita Saxena, Deepak Kumar Jain, Ananya Singhal, "Hand gesture recognition using an android device", International conference on communication systems, and network technologies, 2014.

[8] Rahat Yasir, Riasat Azim Khan," Two handed hand gesture recognition for Bangla sign language using LDA and
ANN", International Conference on Innovative Mechanisms for Industry Applications 2015.

[9] Khan Mohammad Irteza, Sheikh Md. Masudul Ahsan, Razib Chandra Deb," Recognition of Hand Gesture Using Hidden Markov Model", International Conference on Control, Instrumentation, Communication and Computational Technologies 2014 